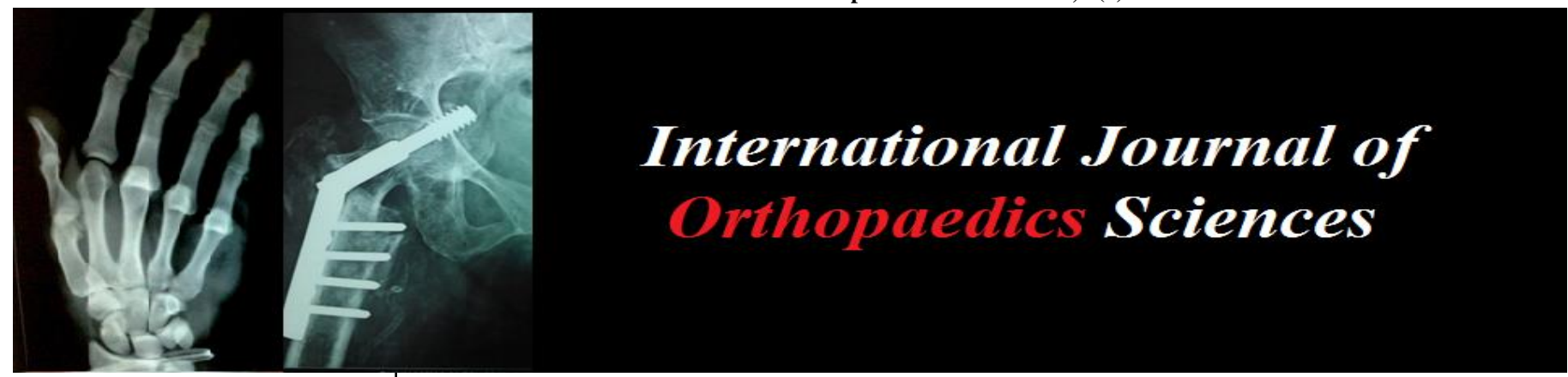

ISSN: $2395-1958$

IJOS 2019; 5(1): 80-82

(C) 2019 IJOS

www.orthopaper.com

Received: 11-11-2018

Accepted: 15-12-2018

Bipul Kumar Garg

Assistant Professor,

Department of Orthopedics,

Grant Medical College and Sir JJ

Group of Hospitals,

Maharashtra, India

Shrikant Pradeep Savant

Senior Resident,

Department of Orthopedics,

Grant Medical College and Sir JJ

Group of Hospitals,

Maharashtra, India

Om Parshuram Patil

Lecturer, Department

Of Orthopedics, Grant Medical

College and Sir JJ Group of

Hospitals, Maharashtra, India

Correspondence

Bipul Kumar Garg

Assistant Professor, Department

Of Orthopedics, Grant Medical

College and Sir JJ Group of

Hospitals, Maharashtra, India

\section{A rare case presentation of eosinophilic granuloma treated with a novel approach: A case report and review of literature}

\section{Bipul Kumar Garg, Shrikant Pradeep Savant and Om Parshuram Patil}

DOI: $\underline{\text { https://doi.org/10.22271/ortho.2019.v5.i1b.18 }}$

Abstract

EG is an uncommon tumor of bone and soft tissue usually presenting in children. It is the bony manifestation of Langerhans cell histiocytosis which shows multisystem involvement.

We report a case of a 4/Male who presented with a painful hip with limp and transtrochanteric tenderness, showing a well-defined osteolytic lesion over the Proximal Femur Metaphysis on XRay with collections of large number of eosinophils mixed with histiocytes on biopsy. Bone Curretage with chemical cauterization and allografting was done for this patient $(1,2)$ which showed relief from symptoms as well as no recurrence in a 1 year follow up.

Keywords: Eosinophilic granuloma, curettage, bone graft, langerhans cell histiocytosis, aneurysmal bone cyst

\section{Introduction}

EG is a benign tumor like condition which is considered to be a part of the spectrum of Langerhans histiocytosis in which cells of the Reticuloendothelial System form granulomatous lesions at microscopic level which grossly appear as osteolytic lesions resembling bone tumors. It commonly affects children (<10 years) ${ }^{[4]}$. The symptoms are - night pains, fever and local tenderness. The Skull is the most common bone involved. EG commonly involves the flat bones $(70 \%)$ due to its tendency of eating up the marrow spaces, also involving the long bones $(30 \%)$ in the diaphyseal region ${ }^{[3]}$.

Radiologically, this disorder can have varied appearances- Vertebra plana is the most common picture. In the diaphyses of long bones, the lesions may have an a permeative appearance with periosteal reactive bone formation ${ }^{[5]}$.

In our study, we report a case of EG in the proximal femur metaphysis in a 4 year old male child with a non-classic presentation, course as well as resolution.

\section{Case Report}

A 4 year old male child presented to the OPD of our hospital with a right sided painful limp since the last four months. Examination indicated tenderness over the right hip - localized over the transtrochanteric area. Range of Motion was normal. XRAY of the Pelvis with Both Hips indicated a cystic, osteolytic type of lesion with regular borders seen in the metaphyseal region of the right proximal femur over the greater trochanter with a good thick posteromedial calcar (Figure-1). X Ray Skull was normal (Figure- 3). MRI revealed a lytic lesion around Proximal Femur showing hypointense to isointense lesion on T1 cuts and hyperintense on T2 cuts (Figure-2) to have conclusive evidence we proceeded with a fluoroscopic guided needle biopsy. The sample obtained was sent to the laboratory for histopathological examination showing- Bony Destruction with collections of large number of eosinophils mixed with histiocytes. The histiocytes showed twisted nuclei suggestive of Langerhans cell histiocytes. The further management planned for the above was - bone curettage with bone grafting (As posteromedial cortical calcar is thick and not involved in Xray) ${ }^{[1,2]}$. Bone Curettage was done followed by chemical cauterization with $10 \%$ Phenol, thorough wash was given and the walls of the cavity were burred off with a diamond burr until fresh bleed was obtained (Figure 4). 
This was followed by allografting. X Ray showed the signs of healing post operatively (Figure-1). There was no recurrence of a similar lesion at the same site/ any other site.
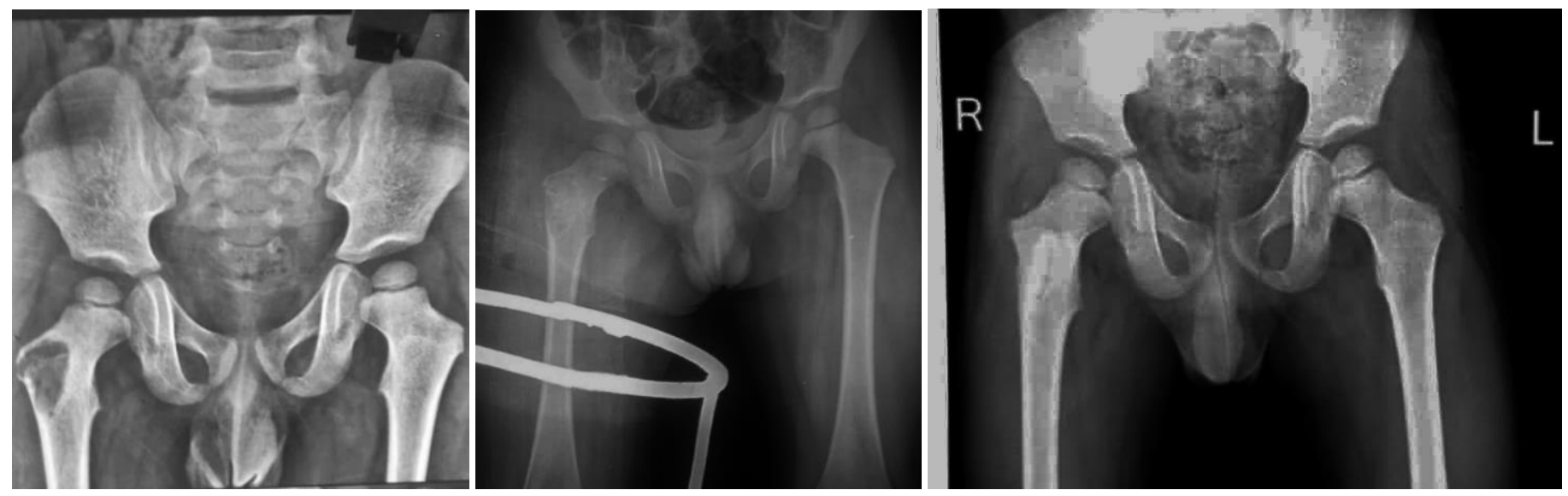

Fig 1: Preoperative, Postoperative and 1 year follow up X rays
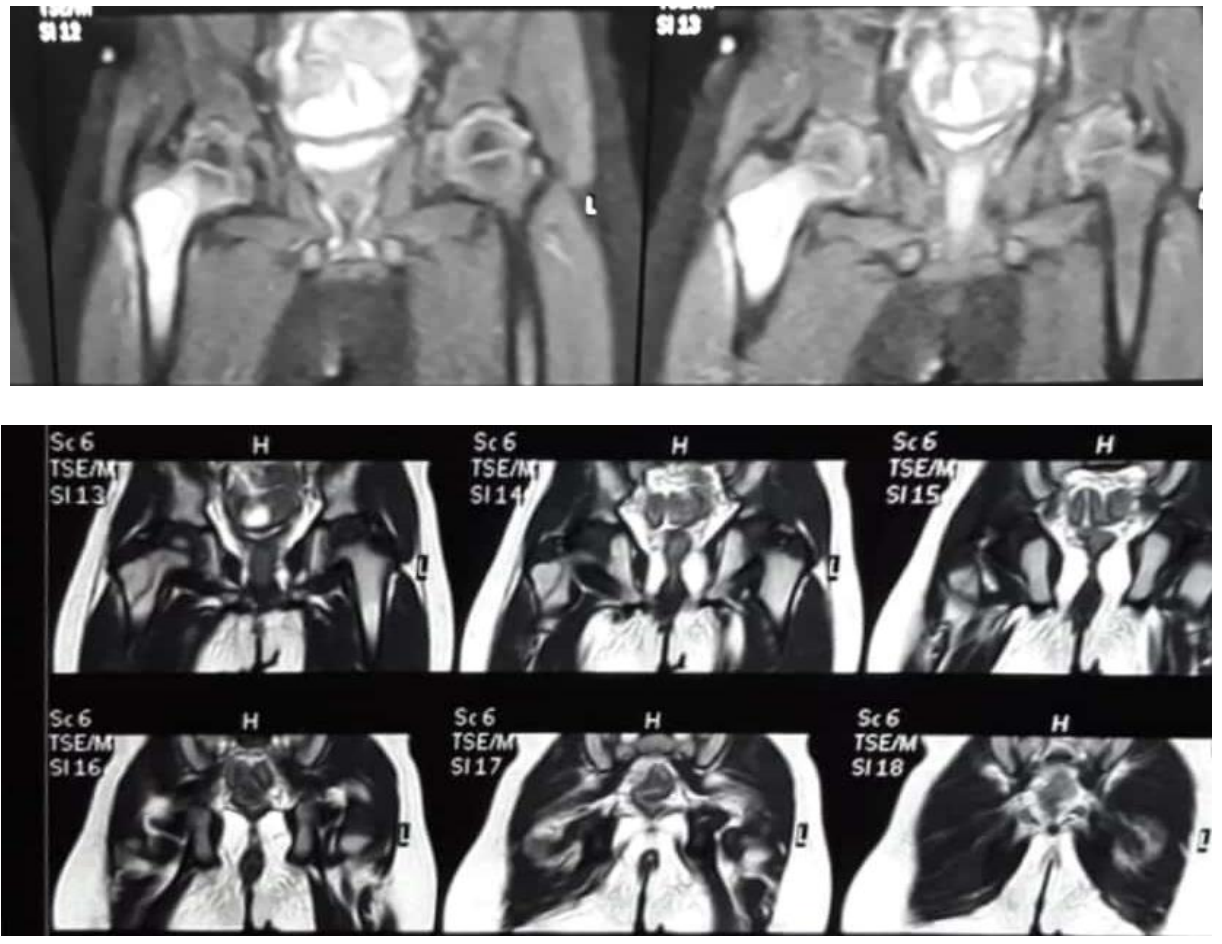

Fig 2: MRI T1 AND T2 Films

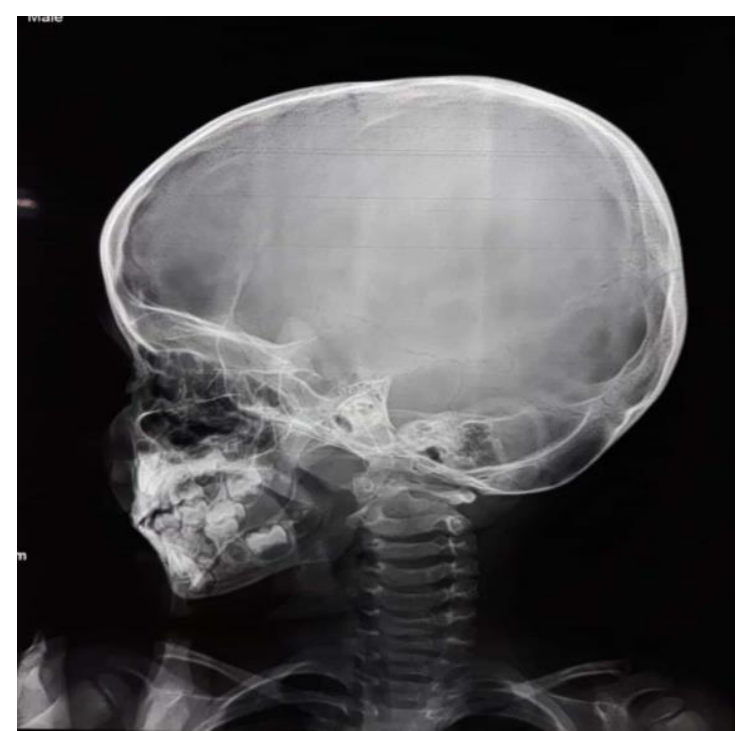

Fig 3: XRay Skull

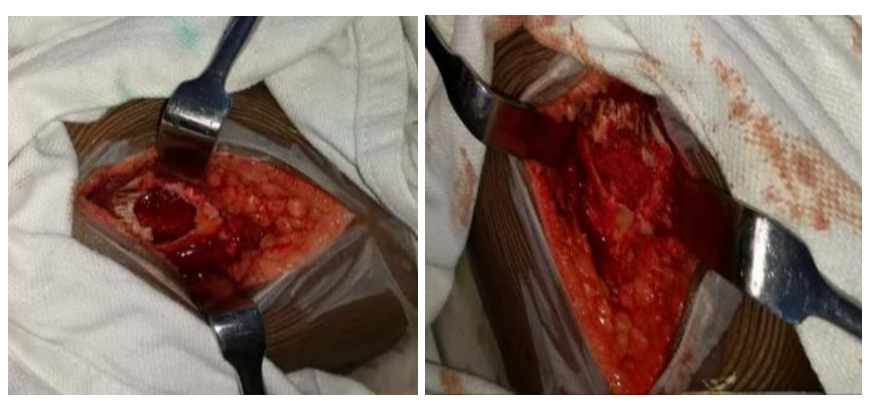

Fig 4: Intra Operative Fresh bleeding cavity and cavity filled with allograft

\section{Discussion}

EG is a rare bone tumour representing $<1 \%$ of all bone tumors with a predilection for males (2:1) (4). EG can be asymptomatic or present as a local swelling with pain and tenderness. No fever or signs of inflammation have been reported. Erythrocyte Sedimentation Rate is raised. Histopathologically, EG is characterized by clonal 
proliferation of Langerhans type histiocytes. These contain Bierbeck granules. The only reliable immunological marker is OKT6 while S-100 protein is usually positive. Investigations include XRay, CT scan and MRI. XRay depicts size and borders. CT and MRI demonstrate the exact size and borders of the tumor as well as the surrounding tissues.

Osteomyelitis, Ewing's sarcoma, Aneurysmal Bone Cyst and Chondroblastoma constitute the differential diagnosis. The treatment options include observation, steroid injections, chemotherapy, irradiation, excision and curettage with or without bone grafting ${ }^{[1,2]}$. In the present case, the patient was symptomatic with a limping gait, and tenderness over the trans trochanteric area of the right hip. Therefore a "wait and watch" strategy could not be applied.

\section{Conclusion}

EG often presents with a wide range of clinical symptoms and sometimes with an unpredictable clinical course requiring multiple surgical as well as non-surgical interventions. We provide additional and independent evidence of the efficacy of bone curettage with chemical cauterization and bone grafting in the management of EG of the femur. This method is also relatively inexpensive with no major side effects as at no point did it include the use of injectable steroids, chemotherapy or irradiation.

\section{References}

1. Postovsky S, Militianu D, Bialik V, Vlodavsky E, Elhasid R, Peled $M$ et al. Concomitant focal fibrocartilaginous dysplasia of the tibia and eosinophilic granuloma of the jaw in a child. J Pediatr Orthop B. 2002; 11(2):172-5.

2. Plasschaert F, Craig C, Bell R, Cole WG, Wunder JS, Alman BA. Eosinophilic granuloma-A Different behavior in children than adults. J Bone Joint Surg Br.

3. Monroc M, Pointe HD, Haddad S, Josset P, Montagne JP. Soft tissue signal abnormality associated with eosinophilic granuloma: correlation of MR imaging with pathologic findings. Pediatr Radiol. 1994; 24:328-332.

4. Ando A, Hatori M, Hosaka M, Hagiwara Y, Kita A, Itoi E. Eosinophilic granuloma arising from the pelvis in children: a report of three cases. Upsala J Med Sci. 2008; 113(1):209-216.

5. Hunter T. Solitary eosinophilic granuloma of bone. J Bone Joint Surg. 1956; 38:545-557. 